\title{
APORTES DE LA CLÍNICA FORENSE PARA CONCEPTUAR EN MATERIA PROBATORIA SOBRE EL RIESGO PARA LA VIDA EN EL DELITO DE TENTATIVA DE HOMICIDIO EN COLOMBIA*
}

\author{
Luis Eduardo Muñoz $P^{a}{ }^{a}$, Liliana Marcela Támara $P .^{b}$ \\ Óscar Armando Sánchez C. ${ }^{c}$, Germán Alfonso Fontanilla D. ${ }^{d}$ \\ Camilo Andrés Castellanos M. ${ }^{e}$, Liliana Dueñas M. ${ }^{f}$, Diego Fernando Gil M. ${ }^{g}$ \\ Laila MH Afifi. ${ }^{h}$, Nancy Fabiola Peña R. ${ }^{i}$, Úrsula Fernanda Castellanos M. ${ }^{j}$
}

Resumen: El establecimiento del tipo penal de tentativa de homicidio exige a la investigación judicial aportar diferentes elementos probatorios. En este sentido, el informe técnico médico-legal de lesiones no fatales, realizado a solicitud de las autoridades, puede aportar valiosos conceptos médicos sobre la gravedad de las mismas, su localización y severidad. Sin embargo, hasta

Fecha de recepción: 13 de noviembre de 2015. Fecha de aceptación: 15 de enero de 2016. Para citar el artículo: MuÑOZ P., LuIS EdUARDo et al. (2015). "Aportes de la clínica forense para conceptuar en materia probatoria sobre el riesgo para la vida en el delito de tentativa de homicidio en Colombia", Revista Derecho Penal y Criminología, Vol. 37, n. ${ }^{\circ} 101$, julio-diciembre de 2015. Bogotá: Universidad Externado de Colombia, pp. 181-202. DOI: http://dx.doi.org/10.18601/ 01210483.v36n101.07

a Médico, Especialización en Gerencia de Riesgos Laborales y Salud en el Trabajo (C). Profesional especializado forense - Grupo de Clínica Forense, Regional Bogotá, Instituto Nacional de Medicina Legal y Ciencias Forenses. Bogotá, D. C., Colombia. Correo-e: lemunoz@medicinalegal.gov.co.

b Médica. Especialista en Bioética. Especialista en Auditoría en Salud. Maestría en Protección Social (C). Profesional especializada forense - Grupo de Clínica Forense, Regional Bogotá, Instituto Nacional de Medicina Legal y Ciencias Forenses. Bogotá, D. C., Colombia. Correo-e: 1tamara@ medicinalegal.gov.co. 
el momento no se han incorporado elementos determinantemente objetivos a partir de la ciencia médica forense para la valoración de la probabilidad que tenga una lesión para causar la muerte. En este artículo se revisan conceptos médico-legales y médicos en el contexto jurídico, que permitirían aportar una ponderación más estructurada de la probabilidad de causar la muerte de una lesión, fundamentalmente a partir de escalas médicas mundialmente reconocidas. Palabras clave: Medicina forense; Homicidio; Heridas y lesiones; Índice de severidad de lesiones.

\title{
CONTRIBUTION TO FORENSIC SCIENCE AIMED TO CONTRIBUTE IN PROBATORY LAW IN THE RISK OF LIFE IN THE CRIME OF ATTEMPTED MURDER IN COLOMBIA
}

\begin{abstract}
The establishment of attempted murder requires the judicial inquiry to provide different evidences. Forensic technical report of nonfatal injuries at the request of the authorities can provide valuable medical concepts on the magnitude, location and severity of the injuries. So far, there has been no objective evidence built from medical science for the assessment of the likelihood of a fatal injury. In this article, we review the forensic and medical-legal concepts, which allow providing percentages of probability of fatal injury from world-renowned medical scales.

Keywords: Forensic Medicine; Homicide; Wounds and Injuries; Trauma Severity Score.
\end{abstract}

c Médico. Especialista forense. Especialista en Docencia Universitaria. Profesor Asociado Universidad Nacional de Colombia. Profesional especializado forense - Grupo de Clínica Forense, Regional Bogotá, Instituto Nacional de Medicina Legal y Ciencias Forenses. Bogotá, D. C., Colombia. Correo-e: osanchez@medicinalegal.gov.co.

d Médico. Profesor de cátedra Pontificia Universidad Javeriana. Profesional especializado forense - Grupo de Clínica Forense, Regional Bogotá, Instituto Nacional de Medicina Legal y Ciencias Forenses. Bogotá, D. C., Colombia. Correo-e: gfontanilla@medicinalegal.gov.co.

e Médico. Magíster en Toxicología. Profesor de cátedra Universidad Militar Nueva Granada Grupo Nacional de Clínica y Odontología Forenses - Subdirección de Servicios Forenses, Instituto Nacional de Medicina Legal y Ciencias Forenses Colombia. Bogotá, D. C., Colombia. Correo-e: camilo. castellanos@medicinalegal.gov.co.

f Médica especialista en Epidemiología. Profesional especializada forense - Grupo de Clínica Forense, Regional Bogotá, Instituto Nacional de Medicina legal y Ciencias Forenses Colombia. Bogotá, D. C., Colombia. Correo-e: lduenas@medicinalegal.gov.co.

g Médico interno. Universidad Nacional de Colombia. Bogotá, D. C., Colombia. Correo-e: dfgilm@ unal.edu.co.

h Médica. Especialista en Salud y Seguridad en el Trabajo. Profesional especializada forense - Grupo de Clínica Forense, Regional Bogotá, Instituto Nacional de Medicina Legal y Ciencias Forenses. Correo-e: malahassan@medicinalegal.gov.co.

i Médica. Profesional universitaria forense - Grupo de Clínica Forense, Regional Bogotá, Instituto Nacional de Medicina Legal y Ciencias Forenses. Bogotá, D. C., Colombia. Correo-e: nancy.pena@ medicinalegal.gov.co.

j Abogada. Especialista en Derecho Público. Magíster en Estudios Urbano-Regionales. Profesora de cátedra Universidad Pontifica Bolivariana. Jueza primera municipal de Piedecuesta. Rama Judicial. Piedecuesta, Colombia. Correo-e: ursula.castellanos@gmail.com. 


\section{INTRODUCCIÓN}

El concepto médico-legal relacionado con la idoneidad de una lesión para producir la muerte ha sido consistentemente utilizado como criterio diferenciador por el juzgador en Colombia, para tipificar un hecho punible, bien como lesiones personales, bien como homicidio en grado de tentativa. Dado el peso probatorio que los informes periciales clínico-forenses tienen para este propósito, es fundamental hacer la presente revisión acerca de los criterios que la clínica forense aporta a dicho concepto.

Es importante advertir que, actualmente, dentro de los lineamientos técnicos del Instituto Nacional de Medicina Legal y Ciencias Forenses de Colombia, no hay una orientación específica en este sentido, siendo entonces el criterio personal de cada médico forense, a partir de su conocimiento técnico-científico y de su experiencia clínica y forense, el que orienta su argumentación. Se hace entonces de imperiosa necesidad revisar la realidad en relación con estos pronunciamientos, trascendentales para agresores y víctimas en el sistema judicial colombiano, y aclarar el uso y la utilidad de emitir conceptos médico-forenses relacionados con la afectación de órganos vitales, riesgo para la vida y lesiones con idoneidad para producir la muerte.

El profesor CÉSAR Augusto GiRALDo (1996) es de los pocos autores forenses nacionales que se ha pronunciado sobre este tema, en términos de objetivación de la severidad de las lesiones y su potencial de letalidad, así como cuando señala:

Lesiones en órganos vitales, como corazón, pulmones, hígado, riñón, estómago, intestino delgado y grueso, páncreas, bazo, vasos de gran calibre (vena cava $\mathrm{y}$ aorta abdominal), si hay tratamiento quirúrgico oportuno puede no presentarse la muerte, así estas lesiones sean idóneas para producir la muerte, y su incapacidad, si el tratamiento es oportuno y adecuado, no rebasa los 60 días.

En otras palabras, las lesiones viscerales anteriores son de naturaleza mortal, y al menos desde el punto de vista de responsabilidad objetiva se comportan como tentativa de homicidio, que no es querellable.

Si el médico examinador en caso de lesiones personales, dando cumplimiento a lo ordenado por el artículo 337 del Decreto 2700 de 1991, señala al final del dictamen un pronóstico de vida, diciendo que las lesiones descritas pusieron en peligro la vida, puede hacer un aporte al funcionario judicial, para enseñarle al menos que desde el punto de vista de la responsabilidad objetiva, deben diferenciarse esas heridas de unas simples lesiones personales que son susceptibles de querella.

Hacer este pronóstico de la naturaleza de las lesiones no parece entrañar un juicio de responsabilidad penal, que le prohíbe la norma al perito (GIRALDO, 1997, p. 67). 
Mencionemos ahora que los oficios petitorios (esto es, las peticiones de las autoridades para las valoraciones médico-legales) incluyen solicitudes como las siguientes:

- Establecer incapacidad médico-legal y si las lesiones causadas afectaron un órgano vital, que pusiera en riesgo la vida del paciente, o hubiese podido causar la muerte.

- 1. Determinar si las lesiones ocasionadas pusieron en riesgo la vida de la víctima, y/o si no hubiese sido por la pronta atención, su vida hubiese corrido peligro 2 . Si las lesiones afectaron órganos vitales.

- Indicar si afectó órganos vitales, y en qué grado se vio comprometida la vida de la víctima, concepto que se requiere con urgencia.

- Dictaminar si las heridas recibidas pusieron en riesgo su vida.

Las conclusiones clínico-forenses, a su vez, pueden ejemplificarse como las siguientes:

- La historia clínica descarta compromiso de órgano vital que hubiera puesto en riesgo la vida del paciente (microcaso: hombre de 35 años ingresado a Hospital de III nivel con herida por arma cortopunzante en región toracoabdominal izquierda anterior, a quien se le practica laparotomía exploratoria con resultado "en blanco").

- De acuerdo con lo consignado en las copias de historia clínica aportadas, no se comprometieron órganos vitales, por suerte, aunque las heridas en zona II de cuello tienen alto riesgo de compromiso de estructuras vasculares y de poner en peligro la vida del paciente (microcaso: hombre de 27 años ingresado a Hospital de III nivel por heridas por botella en cabeza, cara y cuello, requiere cirugía plástica con colgajo local de piel y sutura de lesión del nervio facial, sin compromiso vascular asociado).

- Las lesiones no comprometieron órganos vitales, ni se puso en riesgo la vida del paciente (microcaso: hombre de 54 años ingresado a Hospital de III nivel con amputación de la falange distal de un dedo, posterior a caída de su propia altura tras ser agredido por un tercero).

En una corriente predominante de pensamiento, la amplia proporción de médicos clínicos forenses precisan que sus conceptos médicos no deberían utilizarse para establecer la intencionalidad de un hecho, ante lo cual algunas autoridades responden que es deber de los forenses prestar soporte científico para una adecuada administración de justicia, sin que ello implique conminación a pronunciamiento sobre la intencionalidad de los hechos, y que en efecto deben establecer si hubo compromiso de órgano vital, o si se puso en riesgo la vida del lesionado. 


\section{ALGUNOS CONCEPTOS JURÍDICOS Y NORMATIVAS ALREDEDOR DE LA TENTATIVA DE HOMICIDIO EN COLOMBIA}

Revisados los últimos códigos de Procedimiento Penal colombianos, encontramos lineamientos acerca de la actuación pericial frente a las lesiones. Así, el Decreto 2700 de 1991, en su artículo 337, establecía los parámetros de acción frente al reconocimiento médico en caso de lesiones. También lo hizo la subsiguiente Ley 600 de 2000 sobre los requisitos de la actuación pericial, en el capítulo 3. De igual manera, la actual Ley 906 de 2004, que establece el procedimiento en caso de lesionados o de víctimas de agresiones sexuales en su artículo 250. Sin embargo, es importante resaltar que en ninguno de los códigos se establece que como parte de las conclusiones se debe pronunciar el perito sobre conceptos médicos relacionados con el riesgo para la vida del lesionado, o la afectación de órganos vitales.

No habiendo una clara precisión en relación con el origen y uso de los términos hoy utilizados por funcionarios judiciales y por peritos forenses, se hace necesario establecer algunas precisiones relacionadas con estos conceptos jurídicos y médicos.

En primer lugar, el Código Penal vigente (Ley 599 de 2000) establece en su título III los aspectos relativos a la conducta punible, y en él encontramos descrita la "Tentativa":

\section{Artículo 27 Tentativa}

El que iniciare la ejecución de una conducta punible mediante actos idóneos dirigidos a su consumación, y ésta no se produjere por otras circunstancias ajenas a su voluntad, incurrirá en pena no menor de la mitad del mínimo ni mayor de las tres cuartas partes del máximo de la señalada para la conducta punible consumada.

Cuando la conducta punible no se consuma por circunstancias ajenas a la voluntad del autor o partícipe, incurrirá en pena no menor de la tercera parte del mínimo ni mayor de las dos terceras partes del máximo de la señalada para su consumación, si voluntariamente ha realizado todos los esfuerzos necesarios para impedirla.

De esta manera, la norma amplía el tipo penal, para llegar a la punición de conductas que no finalizan y terminan siendo "delitos frustrados", y para ello tiene en cuenta el peligro inherente corrido por el bien jurídico protegido, específicamente la vida, en la tentativa de homicidio.

Para enunciar lo que se constituye en este delito, se revisan primero los delitos de lesiones y de homicidio, tal como lo establece este mismo Código Penal, así: 
Artículo 103. Homicidio. (Penas aumentadas por el artículo 14 de la Ley 890 de 2004, a partir del $1^{\circ}$ de enero de 2005). El texto con las penas aumentadas es el siguiente: El que matare a otro, incurrirá en prisión de doscientos ocho (208) a cuatrocientos cincuenta (450) meses.

Artículo 111. Lesiones. El que cause daño a otro en el cuerpo o en la salud incurrirá en las sanciones establecidas en los artículos siguientes.

Desde el punto de vista jurídico, la tentativa no está dada para todos los tipos penales; es así como la "tentativa" no es aplicable al delito de lesiones personales, dado que se trata de un delito de resultado, típico, en cuanto dada es la alteración en la integridad física (lesión), y por ende no habría una determinación jurídica objetiva en un paso anterior, esto es, en la mera intención de causar daño, pues los pensamientos e ideas en sí mismos no son punibles.

La sentencia 31948 de 2009 de la Corte Suprema de Justicia retoma la definición de tentativa de Mir PUIG:

El comienzo de ejecución necesario para la tentativa requiere que se traspase la frontera que separa los actos preparatorios (si los hay) de los actos ejecutivos (...). La teoría objetivo-material parte de la necesidad de acudir a un criterio material que permita delimitar objetivamente el inicio de aquel 'campo previo' a la consumación que permite hablar ya de comienzo de la acción típica en sentido amplio (...). En la determinación de cuándo empieza el 'campo previo' en el que ya da comienzo la ejecución debe tomarse en consideración el plan del autor, pero valorándolo desde un prisma objetivo (punto de vista objetivo-subjetivo) (...). Como criterios objetivos de valoración del plan del autor se manejan dos: la puesta en peligro inmediata y la inmediatez temporal. El primer criterio afirma el comienzo de la tentativa cuando se produce ya una inmediata puesta en peligro del bien jurídico, el segundo, cuando se efectúa un acto inmediatamente anterior a la plena realización de todos o algunos de los elementos del tipo. Este segundo criterio ofrece la ventaja de una mayor precisión, pues siempre será discutible cuándo empieza a producirse una puesta en peligro inmediata.

Por otro lado, para el delito de homicidio sí es aplicable el grado de "Tentativa", dado que consiste en un daño objetivo ocasionado por un tercero sin que ocurra el objetivo final de este delito, que es la muerte, y desde el punto de vista jurídico y forense este "daño previo" se refiere al comienzo de la ejecución del delito de homicidio que no llega a consumarse por causas ajenas a la voluntad del autor. Estas son las circunstancias que el administrador de justicia evalúa para penalizar estos hechos previos en grado de tentativa. 
Entonces, el administrador de justicia tiene en cuenta variados elementos para considerar los delitos de homicidio en grado de tentativa, tales como:

- La intención de cometer el delito, circunstancia que según las modalidades de la conducta punible configuran la tentativa como dolosa, es decir, que en los delitos culposos no existe el grado de tentativa. No es posible determinar a otro a la comisión de un delito "eventualmente", así como no es posible iniciar la consumación de un delito mediante actos idóneos e inequívocamente dirigidos a su consumación (tentativa según el CP actual) (EsCOBAR, 2013).

- Principio cogitationis poenam nemo patitur ("nadie sufre penalización por sus pensamientos"). Las ideas no son punibles, por lo que no entran en el concepto de tentativa. Esto se corresponde con la fase subjetiva del iter criminis, que no es objeto del derecho penal.

- El comienzo de la ejecución del acto, parte objetiva del iter criminis, ya sujeto propio del derecho penal, que en el caso de la tentativa determina la no consumación efectiva del delito (en este caso, de homicidio) por circunstancias ajenas al autor.

En este punto del análisis, es fundamental revisar algunos conceptos relacionados con los actos y las conductas que atañen a la modalidad de "Tentativa":

La primera posibilidad es el "resultado fallido", en la cual, circunstancias ajenas al agente activo de la conducta punible impiden el resultado. Es el caso de una persona que de manera deliberada es empujada por otra desde un décimo piso, pero sin embargo cae en una piscina, sufriendo lesiones sin causarse la muerte. El hecho se constituye en una conducta idónea para ocasionar la muerte, pero las circunstancias conexas ajenas al agente no permiten el resultado.

Como segunda, se encuentra el "resultado pendiente", donde el resultado no se ha dado aún, pero de seguir el curso normal se producirá (en este caso, la muerte). Es el caso de unas heridas en el cuello con arma cortopunzante con compromiso de la vía respiratoria o del paquete vascular, en donde el individuo inicialmente queda con vida, pero cuya evolución fisiopatológica, sobre todo de no recibir la atención médico-quirúrgica apropiada y oportuna, tiene altas posibilidades de conducir a la muerte. Así, las acciones de un equipo de salud son las que evitan el resultado, y son la mayoría en las que el juzgador se apoya en un perito forense para su interpretación.

Por último, se encuentran las denominadas “circunstancias inidóneas”, que se refieren a aquellos actos que no tienen la capacidad de generar la muerte de la víctima. Son conductas en las cuales quien perpetra la acción no tiene el conocimiento, la experiencia o la habilidad para producir la muerte como resultado. Estas conductas se dividen en relativas y absolutas; las primeras son aquellas en las cuales el resultado 
se hubiese podido dar en otras circunstancias, como en el caso en el cual se desea matar a una persona y se utiliza el contenido de un frasco rotulado como tóxico, pero cuyo interior no contiene la sustancia prevista y es inocuo su efecto, o en el caso en el cual se tiene una percepción errónea de peligrosidad, como cuando se quiere intoxicar a la víctima con acetaminofén, pensando en que uno de sus efectos adversos puede ser la muerte y es idóneo para causarla. En estos casos de conductas inidóneas relativas, se puede penalizar después de hacer un análisis sobre la peligrosidad de la conducta y con énfasis en la intención criminal.

Por su parte, las conductas inidóneas absolutas son aquellas en las cuales no es posible alcanzar el resultado con las conductas realizadas, lo que se constituye como un delito imposible o irreal. Ejemplo de ello es cuando quien arremete, apuñala con sevicia a quien considera se encuentra debajo de las cobijas, pero solo se trata de objetos materiales. O cuando se le dispara a un cadáver, casos en los cuales no existe el bien jurídico a proteger.

La Sala de Casación Penal de la Corte Suprema de Justicia, en la Sentencia del Proceso 25974 (2007), estudió estos elementos para la configuración de la tentativa a través del análisis de la diferencia entre los actos preparatorios y el principio de ejecución, a partir de las teorías jurídicas subjetivas, las objetivas y las mixtas.

Al respecto, debe precisarse de la mano con lo dicho en un salvamento de voto de la sentencia anteriormente mencionada, que "cualquier exteriorización del agente no puede calificarse como componente del delito, sino solamente aquellas que entrañen una relevante amenaza o lesión al bien jurídico tutelado, y que se conocen como actos ejecutivos". Entonces, hay comportamientos que se pueden confundir con los actos preparatorios, y que si bien tienen relación con el despliegue necesario para la actividad criminal, no alcanzan a ser sancionadas penalmente por no ser relevantes ante la lesión de los bienes jurídicos, excepto se trate de delitos por sí mismos.

En cuanto a la tentativa, es indispensable la determinación de actos en los que se ejecute el delito, o comportamientos idóneos e inequívocamente dirigidos a poner en peligro determinado bien jurídico. En el mismo salvamento de voto al que se hace referencia, el doctor GómEZ QuINTERo expone que

(...) la idoneidad de los actos no es predicable simple y llanamente de los medios utilizados, sino de la conducta objetivamente desplegada por el autor para la consecución del resultado típico. La equivocidad o univocidad hace relación a la aptitud y suficiencia de los actos, según la experiencia, para la producción de la conducta punible.

Entonces, la idoneidad de un comportamiento no se puede confundir con la idoneidad del elemento que se utilizaría para atentar contra algún bien jurídico. Es así como portar un arma blanca no es suficiente para señalar tentativa de homicidio, pese a 
que dicha arma puede causar la muerte, aunque en tal caso también es cierto que del mismo porte no se puede indicar que su objeto sea causar lesiones personales, aunque también pueda hacerlo.

Un ejemplo de lo dicho es que un herido en su región inguinal haya recibido una pronta atención médica que hubiera evitado su muerte, pero que se sepa que el agresor le haya gritado "lo voy a matar"; en este caso, debe tenerse en cuenta que las heridas son propias de una tentativa de homicidio, no solo unas lesiones, ya que no murió por la pronta atención médica y además hubo una manifiesta intención de causar la muerte. La Corte Suprema de Justicia ha dicho que una acción tiene la connotación de tentativa de homicidio si reúne factores como la modalidad del hecho, el medio de agresión empleado, la dirección y el número de golpes y heridas, las condiciones de modo, tiempo y lugar, circunstancias conexas con la acción delictiva; en lo que se refiere al ánimo, es la intención del sujeto, un elemento subjetivo de su conciencia que es de imposible acreditación por prueba directa, que entonces debe inferirse de otros hechos objetivos indiciarios que le permitan al juzgador, de acuerdo con las leyes de la sana crítica, afirmar la existencia de una voluntad dirigida al fin del homicidio, entre ellas las relaciones entre autor y víctima, la personalidad de los mismos y sus actitudes (Sentencia del 21 de junio de 2012. Tribunal Superior de Bogotá. M.P. Alberto Poveda Perdomo).

En el mismo sentido, la Sentencia T-865 de 2014 ratificó que el concepto de tentativa de homicidio exige examinar con detalle los actos producidos por un sujeto sobre otro, su idoneidad para causar daño y las variables que intervinieron para que no se configurara un homicidio, para, finalmente, concluir que se enmarca en una conducta punible denominada tentativa de homicidio. Para esta sentencia, el hecho de tratarse de un accidente con arma de fuego no demuestra, por sí mismo, que se trata de una "tentativa de homicidio". Esto, porque no todos los escenarios en los que está involucrada un arma de fuego son una tentativa de homicidio y, además, porque la tentativa puede suceder en otros contextos sin arma de fuego, así que lo que se debe demostrar es que están presentes los elementos de la conducta punible.

De este modo, doctrinaria y jurisprudencialmente se han establecido dos elementos objetivos para determinar si se está frente a un delito tentado: que exista una progresividad de los actos llevados a cabo por el agresor y que por circunstancias ajenas a la voluntad o decisión de este no se adecuara cabalmente la conducta exteriorizada al tipo objetivo. En todo caso, es la sana crítica del juzgador la que lleva a que con base en las pruebas recaudadas y el análisis de las mismas, se defina si se acepta la imputación, la acusación y la sentencia en la adecuación típica de lesiones personales o en tentativa de homicidio. Esto porque también es cierto que es a la Fiscalía a la que le corresponde hacer la adecuación típica de los hechos, frente a la que el juzgador se debe pronunciar. Además de los elementos objetivos, debe presentarse el elemento subjetivo del dolo, como la voluntad 'finalísticamente' ideada por el agresor para cometer un delito (Corporación Humanas, 2010). Por otro lado, debe 
señalarse que tampoco existe una línea con parámetros previamente establecidos que permitan diferenciar lesiones personales de tentativa de homicidio, pues incluso la jurisprudencia presenta diferencias en salvamentos de voto, por lo cual es necesario que en cada caso se haga un análisis particular con base en todo el acervo probatorio que se recaude en cada caso.

Finalmente, proceder al concepto de la intencionalidad es un criterio de evaluación que compete exclusivamente al juzgador, y para ello hay que hacer una ponderación de la idoneidad de las conductas cometidas y fundamentarse en el propósito o voluntad de darle muerte a una persona. Para establecer la intencionalidad, se tendrán en cuenta los elementos probatorios recopilados durante la fase de investigación, provenientes de la escena, las declaraciones de testigos, las confesiones, los antecedentes del caso y las pruebas periciales.

\section{ÓRGANO VITAL, UNA PERSPECTIVA MÉDICA}

Para el argumento pericial es fundamental la conceptualización del término "órgano vital"; para ello, se parte de la etimología, del latín organum, y del griego

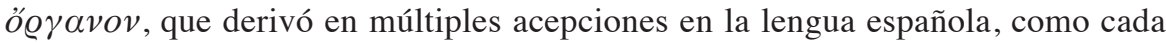
una de las partes del cuerpo animal o vegetal que ejercen una función (RAE, 2014). Ahora bien, desde el enfoque anatómico de la medicina, solo desde VESALIO, en el siglo XVI (GARRISON, 2003), se acuña el término órgano para describir algunas partes organizadas del tejido del cuerpo humano (que ayudan a la nutrición, el cerebro y los órganos de los sentidos). Desde la anatomía humana, se le da el nombre de órgano a partes del cuerpo que colaboran en la realización de una función (JIMÉNEZCastellanos, 2002).

A partir de este concepto anatómico, y dentro del imaginario social, se han identificado como los órganos más importantes: el cerebro, el corazón y los pulmones. Ello se deriva de la percepción que se ha dado, al utilizar términos como "signos vitales" y "soporte vital", y que se han difundido incluso en el mismo personal médico. Si analizamos detenidamente estos conceptos, están enmarcados en la toma de acciones encaminadas a garantizar la función respiratoria, cardiovascular y del sistema nervioso central. Además, en medicina crítica y de urgencias, el enfoque terapéutico se dirige a preservar estas funciones mediante el mantenimiento de un adecuado flujo sanguíneo (Reanimación Cerebro-CardioPulmonar, RCCP) (NADKARNY, 2010).

De esta manera, si bien se puede establecer que estos órganos son esenciales para continuar la vida, la lesión de otros órganos, dependiendo de su magnitud y severidad, finalmente puede ocasionar una alteración sistémica tal que altere la función de los órganos mencionados, como las lesiones sobre estructuras vasculares de grande 
o mediano calibre en cavidad abdominal, pélvica o incluso en las extremidades, así como lesiones sobre la tráquea y sobre la piel (quemaduras extensas). Podría en últimas decirse que todos los órganos que forman parte del organismo humano cumplen una función para la vida, y su alteración puede comprometer el delicado equilibrio que la sustenta.

\section{RIESGO PARA LA VIDA EN MEDICINA}

El último concepto a revisar será el de riesgo para la vida. Riesgo es la probabilidad de que suceda un evento bajo determinadas circunstancias, el cual será inminente en la medida que el tiempo que transcurra convierta el plazo para su verificación en un resultado inmediato. La superación del evento depende de las acciones que contrarresten o eliminen el riesgo. En este sentido, el riesgo para la vida dependerá de la capacidad intrínseca que tenga el daño para conducir a la muerte a un sujeto y las condiciones para superar el riesgo podrán estar determinadas en gran medida por la actuación médica que se logre desplegar.

Como mecanismos para objetivar la gravedad de la lesión, sobre todo en términos de cuantificar en alguna medida su capacidad para producir la muerte, desde la perspectiva técnico-científica se han creado diferentes instrumentos, entre los cuales quisiéramos destacar los siguientes:

Índice de gravedad de las quemaduras (Olguín, 2007; CASTILlo, 2003):

Se ha utilizado para establecer el riesgo vital que implica una quemadura en un paciente determinado y establece una máxima puntuación para pacientes menores de dos años, si el agente causal es eléctrico, si hay lesiones concomitantes, si hay patología asociada, si se tienen condiciones socioeconómicas deficientes o si hay quemaduras en la vía aérea, además de la sumatoria de porcentajes de cada quemadura. Es así como se definen 5 categorías de gravedad de acuerdo con el puntaje alcanzado: sin riesgo vital, con riesgo vital mínimo que requiere hospitalización, con riesgo vital menor o mayor que el $50 \%$ y con riesgo mayor que el $95 \%$ que requiere unidad de cuidados intensivos para su manejo.

Escalas médicas de trauma para valorar potencial riesgo de muerte:

\section{Escala de coma de Glasgow}

El grupo de Glasgow (TEASDALE, 1974) desarrolló un puntaje de valoración del nivel de conciencia, denominado escala de coma de Glasgow (GCS, por sus siglas en inglés). 
Tabla 1. Escala de coma de Glasgow

\begin{tabular}{lll}
\hline $\begin{array}{c}\text { Respuesta ocular o } \\
\text { apertura palpebral }\end{array}$ & \multicolumn{1}{c}{ Respuesta verbal } & \multicolumn{1}{c}{ Respuesta motora } \\
\hline Sin apertura ocular (1) & Sin respuesta verbal (1) & Sin respuesta motora (1) \\
\hline Al estímulo doloroso (2) & $\begin{array}{l}\text { Sonidos incomprensibles } \\
\text { o guturales (2) }\end{array}$ & $\begin{array}{l}\text { Respuesta anormal en } \\
\text { extensión o descerebración (2) }\end{array}$ \\
\hline Al estímulo auditivo (3) & $\begin{array}{l}\text { Palabras fuera de contexto } \\
\text { (respuesta inapropiada) (3) }\end{array}$ & $\begin{array}{l}\text { Respuesta anormal en flexión } \\
\text { o decorticación (3) }\end{array}$ \\
\hline Espontánea (4) & $\begin{array}{l}\text { Desorientado en alguna de } \\
\text { las 3 esferas (confuso) (4) }\end{array}$ & $\begin{array}{l}\text { Retira ante estímulos } \\
\text { nociceptivos o dolorosos (4) }\end{array}$ \\
\hline & Orientado en 3 esferas (5) & $\begin{array}{l}\text { Localiza estímulos } \\
\text { nociceptivos o dolorosos (5) }\end{array}$ \\
\hline & & $\begin{array}{l}\text { Obedece a órdenes o realiza } \\
\text { movimientos espontáneos (6) }\end{array}$ \\
\hline
\end{tabular}

Fuente: Ministerio de Salud y Protección Social, Colciencias. Guía de práctica clínica para diagnóstico

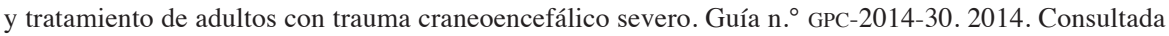
en http:/gpc.minsalud.gov.co/guias/Documents/Trauma\%20Craneoencefalico/GUIA\%20PROFESIONALES_TCE.pdf.

La relación entre el puntaje de Glasgow y la mortalidad se ha revisado ampliamente en diferentes estudios, con diferentes propósitos. La correlación mostrada en la tabla 2 se realiza con propósitos orientativos, cuando se trata de trauma craneoencefálico sin lesiones multisistémicas o cambios fisiológicos mayores. La puntuación de la escala de Glasgow y la mortalidad mostró una relación inversamente proporcional exponencial, con un marcado aumento de la mortalidad en pacientes con puntaje inferior a 9 puntos (ChesNut, 2000). Hay una validación en Argentina sobre esta correlación, con hallazgos similares (PETRONI, 2010). Se puede ampliar la revisión de la literatura en las guías actualizadas sobre pronóstico de trauma craneoencefálico severo e incluir otros indicadores como edad, diámetro pupilar, reflejo fotomotor, hipotensión y hallazgos en imágenes como la Tomografía Axial Computarizada.

Tabla 2. Correlación entre puntaje de Glasgow y mortalidad

\begin{tabular}{ccc}
\hline Puntaje de Glasgow & $\begin{array}{c}\text { Tasa de mortalidad para } \\
\text { trauma craneoencefálico }\end{array}$ & Fuente \\
\hline 3 & $65 \%$ & Fearnside, 1998 \\
\hline $7-13$ & $10-15 \%$ & \\
\hline $3-5$ & $65 \%$ & Generalli, 1982 \\
\hline $6-8$ & $20 \%$ & \\
\hline
\end{tabular}

Fuente: Construida a partir de datos obtenidos de CHESNUT, 2000. 


\section{ESCALA REVISADA DE TRAUMA (RTS)}

Dentro de las escalas de trauma en general se encuentra la RTS (Revised Trauma Score), utilizada en el triage de pacientes en los servicios de urgencias. Ampliamente validada en la literatura y que utiliza el puntaje de la escala de coma de Glasgow, el valor de la tensión arterial sistólica y el valor de la frecuencia respiratoria para establecer cinco categorías (con puntuaciones de 0 a 4) de acuerdo con rangos de valores para cada uno de los ítems evaluados. (CHAMPION, 1981; JAFFE, 1991). El puntaje global ponderado se obtiene a partir de la siguiente fórmula: $\mathrm{RTS}=0.9368$ x GCS (puntaje para la escala de coma de Glasgow) +0.7326 x SBP (puntaje para la presión arterial sistólica) $+0.2908 \times \mathrm{RR}$ (puntaje para la frecuencia respiratoria).

Tabla 3. Puntaje de Trauma Revisado (RTS) por ítems

\begin{tabular}{cccc}
\hline $\begin{array}{c}\text { Escala de coma de } \\
\text { Glasgow (GCS) }\end{array}$ & $\begin{array}{c}\text { Presión arterial } \\
\text { sistólica (SBP) }\end{array}$ & $\begin{array}{c}\text { Frecuencia } \\
\text { respiratoria (RR) }\end{array}$ & Puntaje \\
\hline $13-15$ & $>89$ & $10-29$ & 4 \\
\hline $9-12$ & $76-89$ & $>29$ & 3 \\
\hline $6-8$ & $50-75$ & $6-9$ & 2 \\
\hline $4-5$ & $1-49$ & $1-5$ & 0 \\
\hline 3 & 0 & 0 & 1 \\
\hline
\end{tabular}

Fuente: Modificada de Trauma.org (2015).

Entonces, el puntaje final total está en el rango de 0 a 7,8408. El propósito fundamental es precisar un umbral de puntaje menor que 4 para identificar los pacientes que deben ser manejados en un centro de trauma, y establecer una correlación de probabilidades de sobrevida, como se aprecia en la gráfica.

\section{Gráfica 1. Probabilidad de sobrevida de acuerdo con el RTS}

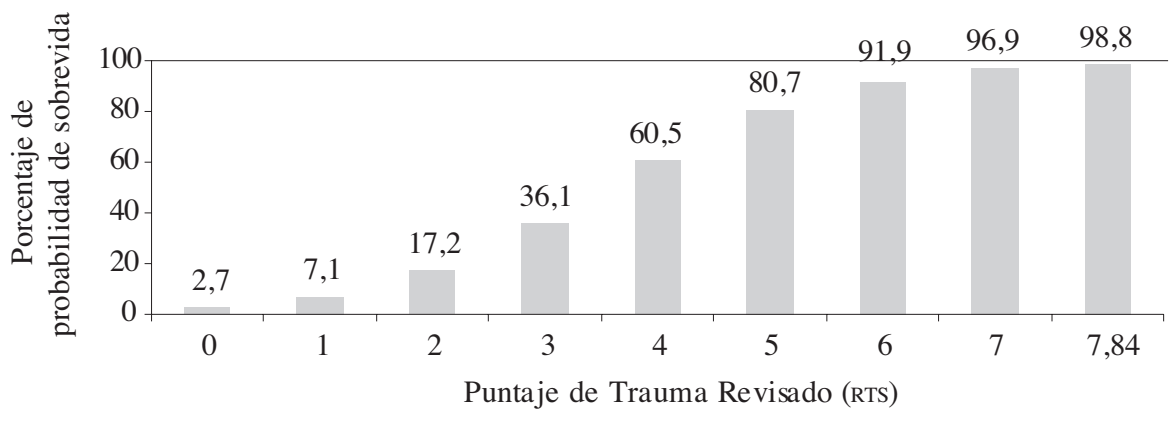

Fuente: Construido a partir de Levy $(1978,1982)$ y CHAMPION (2002). 


\section{ESCALA ABREVIADA DE LESIONES}

La escala abreviada de lesiones (AIS, Abbreviated Injury Scale) fue propuesta en 1969 por la Asociación para el Avance de la Medicina Automovilística y ha sido revisada desde 1990. Actualmente, la versión de 2008 está disponible mediante licenciamiento en diferentes instituciones de varios países desarrollados del mundo. Es la base para múltiples escalas de trauma, con diferentes propósitos, entre otros, unificar los criterios de clasificación de las lesiones con respecto a su localización y severidad, y en términos estadísticos permitir correlaciones entre las distintas condiciones de los traumas y los desenlaces finales, muerte, recuperación, entre otros (AAAM, 2015). Ha sido utilizada también con propósitos forenses, como una forma de describir y analizar las lesiones post mortem (ZVULUM, 1996; VERNARD, 1998).

En la tabla 4 se aprecia el puntaje de severidad de las lesiones de 1 a 6 para cada lesión. Se encuentra una clasificación para lesiones generales y luego clasificaciones por regiones anatómicas (general; cara, cabeza y cuello; pared torácica, lesiones cardíacas, pulmonares, vasculares torácicas, diafragmáticas, hepáticas, del árbol biliar extrahepático, pancreáticas, esofágicas, gástricas, duodenales, de intestino delgado, colónicas, rectales, vasculares abdominales, adrenales, renales, ureterales, vesicales, uretrales, uterinas en el embarazo, trompa de Falopio, ováricas, vaginales, vulvares, testiculares, peneanas, vasculares periféricas, extremidades y pelvis), y se presenta el ejemplo de las lesiones uterinas durante el embarazo.

Tabla 4. Severidad de las lesiones (AIS)

\begin{tabular}{lc}
\hline \multicolumn{1}{l}{ Severidad de las lesiones } & Puntaje \\
\hline Lesión menor & 1 \\
\hline Lesión moderada & 2 \\
\hline Severa pero no amenaza la vida & 3 \\
\hline Amenaza potencialmente la vida pero es probable sobrevivir & 4 \\
\hline Crítico con sobrevida incierta & 5 \\
\hline Lesión sin posibilidades de sobrevivir (máxima posible) & 6 \\
\hline Severidad desconocida & 9 \\
\hline \multicolumn{1}{c}{ Lesiones uterinas en embarazo } & 2 \\
\hline I Contusión o hematoma (sin abruptio) & 3 \\
\hline II Laceración superficial (<1cm) o abruptio placentario parcial & 3 \\
\hline $\begin{array}{l}\text { III Laceración profunda } 1 \text { o más cm en el segundo trimestre o abruptio } \\
\text { placentario }>25 \% \text { pero }<50 \%\end{array}$ & 4 \\
\hline III Laceración profunda 1 o más cm en primer trimestre & 4 \\
\hline IV Laceración uterina 1 o más cm con $>50 \%$ de abruptio placentario & 4 \\
\hline V Ruptura uterina segundo semestre & \\
\hline
\end{tabular}




\begin{tabular}{lc}
\hline v Ruptura uterina tercer trimestre & 5 \\
\hline v Abruptio placentario completo & $4-5$ \\
\hline Aumentar un grado para lesiones múltiples por encima de grado III & \\
\hline
\end{tabular}

Fuente: Adaptada de AAST y Traumascore.org.

\section{Puntaje de severidad de las lesiones}

La calificación de los puntajes de las lesiones por regiones anatómicas antes descrito fue el punto de partida para los cálculos realizados por BAKER (1974), desde los cuales se estableció la escala de gravedad de las lesiones (ISs, Injury Severity Score).

Para obtener la valoración de esta escala, se deben identificar los mayores puntajes obtenidos para las lesiones en cada una de las distintas siete regiones corporales de la AIs, de los cuales se escogen los tres más altos y cada uno se eleva al cuadrado. Si a una lesión se le asigna un puntaje de 6 (lesión que no permite la sobrevida), automáticamente se asigna al Iss un valor de 75 . El cálculo se realiza así:

ISS $=(\text { mayor puntaje de AIS })^{2}+(\text { segundo mayor puntaje de AIS })^{2}+($ tercer mayor puntaje de AIS) ${ }^{2}$.

El resultado clasifica a la persona lesionada en un puntaje entre 0 y 75 puntos, valor que, asociado a factores como la edad, permitirá establecer una tasa de mortalidad a corto plazo (ver tabla 5). Se han validado diferentes versiones del instrumento para estimar la probabilidad de muerte al ingreso, dependiendo del estado hemodinámico y de la gravedad de la lesión anatómica (Sacco, 1999; Gelves, 2009; Goris, 1983).

Tabla 5. Tasa de mortalidad de acuerdo a ISS

\begin{tabular}{cccc}
\hline Puntaje & $\begin{array}{c}\text { Tasa de mortalidad } \\
\text { si }<\mathbf{4 9} \text { años }\end{array}$ & $\begin{array}{c}\text { Tasa de mortalidad } \\
\text { si } \mathbf{5 0 - 6 9} \text { años }\end{array}$ & $\begin{array}{c}\text { Tasa de mortalidad } \\
\text { si }>\mathbf{7 0} \text { años }\end{array}$ \\
\hline 5 & 0 & 3 & 13 \\
\hline 10 & 2 & 4 & 15 \\
\hline 15 & 3 & 5 & 16 \\
\hline 20 & 6 & 16 & 31 \\
\hline 25 & 9 & 26 & 44 \\
\hline 30 & 21 & 42 & 65 \\
\hline 40 & 47 & 62 & 100 \\
\hline 45 & 61 & 67 & 100 \\
\hline 50 & 75 & 83 & 100 \\
\hline 55 & 89 & 100 & \\
\hline
\end{tabular}

Fuente: Construida a partir de BAKER (1974). 
Se notó que elegir solo una de las lesiones por región corporal podía sesgar el resultado, por lo cual se propuso una modificación, el Nuevo Puntaje de Severidad de las Lesiones (NISS, por sus siglas en inglés), que se calcula con la suma de los cuadrados de los tres puntajes mayores independientemente de la región corporal que afecten, lo cual ha demostrado superiores correlaciones estadísticas (BAKER, 1997).

La crítica más fuerte a esta evaluación era la falta de precisión de la clasificación con las lesiones encontradas en los casos. Dicha crítica ha sido progresivamente corregida en la medida que se han precisado las lesiones y se han obtenido bases de datos cada vez mayores. Sin embargo, es fundamental contar con el licenciamiento de la Escala Abreviada de Lesiones para su aplicabilidad de manera formal.

Existen otros puntajes, como el CRAMS (Circulation, Respiration, Abdomen, Motor, Speech), utilizado para evaluar pacientes que pueden requerir transporte a un centro hospitalario para manejo del trauma (Gormincan, 1982; Clemmer, 1985); el Índice de Trauma Penetrante Abdominal (PATI), el Puntaje de Trauma y de Severidad Lesional (TRISS), la Caracterización de la Severidad del Trauma (ASCOT), el Índice de Trauma Hospitalario (HTI), y la evaluación fisiológica aguda y de salud crónica (APACHE), entre otros. Hay validaciones y comparaciones colombianas de algunas de estas escalas para evaluar pronóstico vital, llegando en algunos tipos de trauma a consensos, independientemente de la escala, como el caso de los traumas neurológicos (CAMARGO, 2003) o de traumas penetrantes abdominales (GELVES, 2009).

\section{DISCUSIÓN}

En medicina clínica forense, especialmente en el contexto del sistema penal colombiano vigente, en el marco de la normativa que contempla la tentativa de homicidio como aquella circunstancia no consumada de un daño corporal que en su intencionalidad tendría al cegamiento de la vida, por ende del bien jurídico lesionado, y en el contexto de una estructura probatoria objetiva que requiere la materialización científica de la argumentación jurídica del hecho punible, es crucial contar con conceptos claros y herramientas sustentables y reproducibles para la experticia médico legal.

Tradicionalmente, y hasta la fecha, no han existido sustanciales avances en términos de normalización de conceptos, en el terreno de lo médico-forense, acerca del riesgo a la vida y la letalidad de las lesiones; es quizás, entonces, una oportunidad para proponer el estudio de estas cuestiones, empezando por precisar de manera definida y unificada los conceptos que les componen, y cómo se definen, invitando desde este espacio al sector judicial a la reflexión y esfuerzos conjuntos con la medicina legal para el desarrollo científico en aras de una delimitación integral de lo que significa solicitar conceptos médicos forenses relacionados con la tentativa de homicidio. 
Es evidente, a través de nuestra experiencia clínica forense, la tendencia de la parte acusadora de solicitar conceptos médico-forenses para apuntalar la tipificación del delito de homicidio en grado de tentativa. Los conceptos solicitados giran alrededor de "riesgo a la vida", "lesión de órgano vital", "efectos que hubiese tenido la no pronta atención”, elementos que, en efecto, son de importante análisis y consideración por cuanto hablan, en cada caso en particular, de la localización y severidad de las lesiones, de su evolución fisiopatológica y de su potencial inmanente de letalidad. Sin embargo, se debe recordar que son solo una parte del constructo típico de la conducta punible, donde desde el punto de vista médico-legal se recomienda avanzar en el análisis de la letalidad intrínseca que entrañan ciertos mecanismos de lesión (v.g., las armas cortopunzantes, los proyectiles de arma de fuego), aun cuando exista indemnidad de órganos vitales y, por supuesto, la intencionalidad del agente y la fase objetiva del iter criminis.

De esta forma, siendo en todo caso de gran importancia los elementos que aporta la experticia clínica forense, se propone que en Colombia, en el hoy de un sistema penal de prueba objetiva, oralidad, sana contradicción e igualdad de oportunidades, y en el cual es crucial la ponderada valoración con diáfano criterio por parte del juzgador, debe avanzarse en el terreno de la objetivación médica del riesgo a la vida. Para ello, se propone la utilización de instrumentos de medición de severidad del trauma, particularmente aquellos que, a través de estudios con alto rigor científico, han precisado correlación con tasas de mortalidad. El uso de estos instrumentos exige refinar la descripción de las lesiones a niveles de mayor detalle anatómico-patológico en el registro en las historias clínicas. Es de resaltar que estas herramientas deben ser aplicadas de acuerdo con las particularidades de cada caso, y analizadas a la luz de las circunstancias clínicas de cada examinado.

Este es un punto de partida para revisiones jurídicas amplias sobre el concepto de tentativa, la necesidad de los conceptos científicos clínico-forenses, la idoneidad de los registros médicos, de los exámenes paraclínicos, de las imágenes diagnósticas e, incluso, de los registros de atenciones prehospitalarias y la utilización de instrumentos como los propuestos. Todo esto en el favorecimiento de una resolución más oportuna y ágil de los procesos judiciales, propendiendo así a una justicia más eficaz.

\section{BIBLIOGRAFÍA}

AAAM - Association for the Advancement of Automotive Medicine (2015). About AIS. Disponible en http://www.aaam.org/about-ais.html.

AAST - American Association for the Surgery of Trauma (2015). Trauma Source. Injury Score Scale. Disponible en http://www.aast.org/library/traumatools/injuryscoringscales.aspx\#cervical. 
Adams, V. y Carrubba, C. (1998). "The Abbreviated Injury Scale: Application to Autopsy Data”, en Am J Forensic Med Pathol. Vol. 19, n. ${ }^{\circ} 39$, pp. 246-251.

BAKer, S. P. y O’Neill B, et al. (1974). “The Injury Severity Score: A Method for Describing Patients with Multiple Injuries and Evaluating Emergency Care", en $J$ Trauma. Vol 14, pp. 187-196.

BAKer, S. P. (1997). “Advances and Adventures in Injury Prevention”, en J Trauma: Injury Infect Crit Care. Vol 42, pp. 369-73.

CAmargo, R. D. (2003). “Comparación de índices pronósticos de morbimortalidad en pacientes neurológicos", en Revista Academia Nacional de Medicina. Vol. 25, n. ${ }^{\circ}$ 1, p. 61. Disponible en http://encolombia.com/medicina/revistas-medicas/academedicina/va-61/academ25161-junta/.

Castillo, P. (2003). "Quemaduras. Conceptos para el médico general”, en Cuad. Cir. Vol 17,pp.58-63. Disponible en http://mingaonline.uach.cl/pdf/cuadcir/v17n1/ art10.pdf

Champion, H. R. (2002). “Trauma Scoring”, en Scandinavian Journal of Surgery. Vol. 91, pp. 12-22. Disponible en http://sjs.sagepub.com/content/91/1/12.full.pdf+html.

Champion, H. R., Sacco, W. J. et al. (1989). “A Revision of the Trauma Score”, en J Trauma. Vol. 29, pp. 623-629.

Chesnut, R. M.; Ghajar, J.; MaAs, A.I.R.; Marion, D. W.; Servadei, F.; Teasdale, G.M. y Beverly, C.W. (2000). "Early Indicators of Prognosis in Severe Traumatic Brain Injury". Brain Trauma Foundation. TBI Guidelines. Disponible en https://www. braintrauma.org/pdf/protected/prognosis_guidelines.pdf.

Clemmer, T. P.; Orme, J. F. Jr. et al. (1985). "Prospective Evaluation of the CRAMS Scale for Triaging Major Trauma”, en J Trauma. Vol. 25, pp. 188-191.

Congreso de la República de Colombia (24 de julio de 2000). "Ley 600. Por la cual se expide el Código de Procedimiento Penal”, en Diario Oficial de la República de Colombia, 44.097. Bogotá. Disponible en http://www.secretariasenado.gov.co/ senado/basedoc/ley_0600_2000.html.

Congreso de la República de Colombia (1 de septiembre de 2004) "Ley 906. Por la cual se expide el Código de Procedimiento Penal”, en Diario Oficial de la República de Colombia, 45.658. Bogotá. Disponible en http://www.secretariasenado.gov. co/senado/basedoc/ley_0906_2004.html. 
Congreso de la República de Colombia (24 de julio de 2000) "Ley 599. Por la cual se expide el Código Penal”, en Diario Oficial de la República de Colombia, 44.097, Bogotá. Disponible en http://www.secretariasenado.gov.co/senado/basedoc/ ley_0599_2000.html.

Copes, W. S.; Sacco, W. J.; Champion H. R. y Bain, L. W. (1989). "Progress in Characterising Anatomic Injury", en Proceedings of the $33^{\text {rd }}$ Annual Meeting of the Association for the Advancement of Automotive Medicine. Baltimore, MA, USA, pp. 205-218.

CORPORACIÓN HumANAS (2010). Estudio de la jurisprudencia colombiana en casos de delitos sexuales cometidos contra mujeres y niñas. Ediciones Antropos. Disponible en http://www.humanas.org.co/archivos/estudio_de_la_jurisprudencia.pdf.

Corte Constitucional. Sentencia T-865 de 2014. Disponible en http://www.corteconstitucional.gov.co/relatoria/2014/T-865-14.htm.

Corte Suprema de Justicia. Sala de Casación Penal, proceso 31948, sentencia del 27 de julio de 2009, citando a MiR PUIG (sf). Disponible en http://www.cispa.gov. co/index.php?option=com_docman\&task=doc_download\&gid=573\&Itemid=24 .

Corte Suprema de Justicia. Sala de Casación Penal, proceso 25974, sentencia del 8 de agosto de 2007.

Escobar Behar, V. y Monsalve Correa, S. (2013). El dolo eventual en la jurisprudencia de la Corte Suprema de Justicia: periodo 1980-2011. Universidad de Antioquia. Disponible en https://aprendeenlinea.udea.edu.co/revistas/index.php/ red/article/download/20033/16927.

Garrison, D.; Hast, M., y Northwestern University. (2003). Andrea Vesalii De Humani Corporis Fabrica. Preface. Versión Online. Disponible en http://vesalius. northwestern.edu/.

Gélvez, S.; Ordóñez, C.; Badiel, M.; Ramírez, Ó.; Pino, L.; García, A.; Granados, M.; Ospina, G.; Peitzman, A. y Puyana, J. (2009). "Evaluación de las escalas ISS y NISS en trauma penetrante grave", en Revista Colombiana de Cirugía. Vol. 24, n. ${ }^{\circ}$ 4, 2009, pp. 229-235. Disponible en http://www.scielo.org.co/scielo. php?script=sci_arttext\&pid=S2011-75822009000400004\&lng=en\&tlng=es. Consultado el 19 de mayo de 2015.

Giraldo, C.A. (1996). Medicina Forense. Medellín: CIB. 8. a ed. Capítulo v. Lesiones Personales, p. 67. 
Goris, R. J. A. (1983). "The Injury Severity Score”, en World JSurg. Vol. 7, n. ${ }^{\circ}$, pp.12-18. Disponible en http://www.acrm.org.my/ntrd/documents/literatures/1983.\%20The\%20Injury\%20Severity\%20Score.pdf.

Gormican, S.P. (1982). “CRAms Scale: Field Triage of Trauma Victims”, en Ann Emerg Med. Vol. 11, n. 3, pp.132-135.

Hazinski, M. F.; Nolan, J.P.; Billi, J. E.; Böttiger, B. W.; Bossaert, L.; DE CAen, A. R.; Deakin, C. D.; Drajer, S.; Eigel, B.; Hickey, R.W.; Jacobs, I.; Kleinman, M. E.; Kloeck, W.; Koster, R. W.; Lim, S. H.; Mancini, M. E.; Montgomery, W. H.; Morley, P. T.; Morrison, L. J.; Nadkarni, V. M.; O’Connor, R. E.; OKada, K.; Perlman, J. M.; Sayre, M. R.; Shuster, M.; Soar, J.; Sunde, K.; Travers, A. H.; Wyllie, J. y Zideman, D. (2010). "Part 1: Executive Summary: 2010 International Consensus on Cardiopulmonary Resuscitation and Emergency Cardiovascular Care Science with Treatment Recommendations", en Circulation. Vol. 122, (suppl 2):S250-S275.

JAFFE D. y Wesson, D. (1991). "Emergency Management of Blunt Trauma in Children”, en N Engl J Med. Vol. 324, 19911477-1482. Disponible en http://www.nejm. org/doi/full/10.1056/NEJM199105233242106.

Jiménez-Castellanos, J.; Catalina, C. J. y Carmona, A. (2002). Lecciones de neuroanatomía clínica. Sevilla, España: Universidad de Sevilla. p. 22.

Levy, P. S.; Goldberg, J.; Rothrock, J. (1982). “The Revised Estimated Survival Probability Index of Trauma Severity", en Public Health Report. Vol. 97, n. ${ }^{\circ}$, pp. 452-459. Disponible en http://www.ncbi.nlm.nih.gov/pmc/articles/PMC1424362/ pdf/pubhealthrep00115-0058.pdf.

Levy, P.S.; Mullner, R.; Goldberg, J. y Gelfand, H. (1978). “The Estimated Survival Probability Index of Trauma Severity", en Health Service Research. Disponible en http://www.ncbi.nlm.nih.gov/pmc/articles/PMC1072028/pdf/hsresearch00542-0030.pdf.

Ministerio de Salud y Protección Social, Colciencias (2014). Guía de práctica clínica para diagnóstico y tratamiento de adultos con trauma craneoencefálico severo. Guía $n .{ }^{\circ}$ GPC-2014-30. Disponible en http://gpc.minsalud.gov.co/guias/Documents/ Trauma\%20Craneoencefalico/GUIA\%20PROFESIONALES_TCE.pdf.

Moore, E. E.; Cogbill, T. H.; Malangoni, M.; Jurkovich, G. J. y Champion, H. R. "Scaling System for Organ Specific Injuries", en ASST American Association for the Surgery of Trauma. Trauma Source. Injury Score Scale. Disponible en http://www. aast.org/library/traumatools/injuryscoringscales.aspx\#cervical. 
Nadkarni, V. M.; Nolan, J. P.; Billi, J. E.; Bossaert, L.; Böttiger, B. W. y ZideMAN, D. (2010). “2010 International Consensus on Cardiopulmonary Resuscitation and Emergency Cardiovascular Care Science with Treatment Recommendations: Part 2: International Collaboration in Resuscitation Science: 2010 International Consensus on Cardiopulmonary Resuscitation and Emergency Cardiovascular Care Science With Treatment Recommendations", en Circulation, n. ${ }^{\circ} 122,2010$, pp.S276-S282, doi:10.1161/CIRCULATIONAHA.110.970921. Disponible en https://circ.ahajournals. org/content/122/16_suppl_2/S276.full.

Olguín, F.; Rivera, R.; Brunser, O.; Olguín, R. y GotTeland, M. (2007). “Estudio de la función de barrera gástrica e intestinal y su evolución en el tiempo en pacientes quemados", en Cir.plást.iberolatinoam [revista en la Internet]. Vol. 33, n. ${ }^{\circ}$ 4, pp. 203 207. Disponible en http://scielo.isciii.es/scielo.php?script=sci_arttext\&pid=S0376$78922007000400001 \& \operatorname{lng}=$ es. Consultado el 15 de mayo de 2015.

PAlmer, C.S. y Franklin, M. (2011). “Assessment of the Effects and Limitations of the 1998 to 2008 Abbreviated Injury Scale Map Using a Large Population-Based Dataset", en Scandinavian Journal of Trauma, Resuscitation and Emergency Medicine. Vol. 19, n. ${ }^{\circ}$ 1. doi:10.1186/1757-7241-19-1. Disponible en http://www.sjtrem. com/content/19/1/1.

Petroni, G.; Quaglino, M.; Lujan, S.; Kovalesky, L.; Rondita, C.; VidetTa, W. y Chesnut, R. (2010). "Early Prognosis of Severe Traumatic Brain Injury in an Urban Argentinian Trauma Center”, en J Trauma. Vol. 68, pp. 564-570. Disponible en http://www.globalneurotrauma.org/articles/EarlyPrognosisofSevereTBI_2010.pdf.

Presidente de la República de Colombia (30 de noviembre de 1991). "Decreto n. ${ }^{\circ}$ 2700. Por medio del cual se expiden y se reforman las normas de procedimiento penal", en Diario Oficial de la República de Colombia, 40.190. Bogotá, Colombia. Disponible en ftp://ftp.camara.gov.co/camara/basedoc/codigo/codigo_procedimiento_penal_1991.html.

Real Academia Española, RAE. Diccionario de la lengua española. Edición 23. Disponible en http://buscon.rae.es/drae/.

SACCO W. J. et al. (1999). "Comparison of Alternative Methods for Assessing Injury Severity Based on Anatomic Descriptors", en J Trauma. Vol. 47, n. 3, pp. 441-446; discussion 446-7. Disponible en http://www.ncbi.nlm.nih.gov/pubmed/10498295.

TeAsdale G y JennetT, B. (1974). “Assessment of Coma and Impaired Consciousness. A Practical Scale”, en The Lancet. Vol. 13, n. 2 (7872), 1974: 81-4. Disponible en http://www.compassionandsupport.org/pdfs/professionals/molst/glasgow_coma.pdf. 
Trauma.org. (2015). Revised Trauma Score. Disponible en http://www.trauma.org/ archive/scores/rts.html.

Trauma scores. Injury Severity Score. Disponible en http://www.traumascores .com/ index.php/scores2/16-allgemein/105-104.

Zvulum F.; Chen, K.; Jehuda, H.; Baruch, M.; Michael, S. y Shmuel, S. (1996). "The Abbreviated Injury Scale: A Valuable Tool for Forensic Documentation of Trauma", en Am J Forensic Med Pathol. Vol. 17 n. o 3, pp. 233-238. Disponible en http://journals.lww.com/amjforensicmedicine/Abstract/1996/09000/The_Abbreviated_Injury_Scale__A_Valuable_Tool_for.11.aspx. 